\title{
Towards Cell-based Therapeutics: A Bio-inspired Autonomous Drug Delivery System
}

\author{
Chieh Lo ${ }^{\mathrm{a}, *}$, Kartikeya Bhardwaj ${ }^{\mathrm{a}}$, Radu Marculescu ${ }^{\mathrm{a}}$ \\ ${ }^{a}$ Department of Electrical and Computer Engineering, Carnegie Mellon University, 5000 Forbes Ave, Pittsburgh, PA, 15213
}

\begin{abstract}
Traditional drug delivery systems are often inefficient and imprecise as they mainly rely on diffusion processes. For example, the blood circulation system is often used to transport and deliver drugs throughout the body; due to the randomness involved in molecular diffusion, this approach can cause inefficient and imprecise delivery of drugs. To address this problem, we propose an autonomous and adaptive bacteria-based drug delivery system that integrates bacterial chemotaxis and quorum sensing in order to deliver drugs efficiently and precisely at various location in the human body. More specifically, we design a synthetic AND gate that enables bacteria to detect molecules produced by tumors and release the appropriate drugs in a coordinated manner; the system can also dynamically adjust the amount of drugs released based on tumor size and activity level. Our experimental results show that the proposed system can be effectively used for cell-based therapeutics while preventing drug overuse and multi-drug resistance.
\end{abstract}

Keywords: Drug delivery, Cell-to-cell communication, Molecular communication

\section{Introduction}

Traditional drug delivery systems like injecting drugs into blood vessels are often inefficient as they rely on diffusion processes to deliver drugs. Indeed, diffusion makes these systems imprecise spatially (i.e., with respect to a target location) and in terms of dosage [1]; this can lead to drug overuse and multi-drug resistance $[2,3]$. To address both problems, targeted cellbased therapies have gained significant attention recently [4]. This type of treatment interferes directly with specific cell molecules required for tumor growth, rather than indiscriminately targeting malignant and nonmalignant cells as is the case in traditional chemotherapy [5]. For instance, Alexander-Bryant et al. introduce strategies for designing targeted cancer therapies in [6]. More precisely, they propose to deliver a high dose of anticancer drugs directly to the cancer tumor, while minimizing the drug uptake by nonmalignant cells. However, the problem of how to efficiently deliver the drugs to the target location remains unsolved.

In recent years, targeted drug delivery has been actively studied and a number of mathematical models

\footnotetext{
${ }^{*}$ Corresponding author

Email addresses: chiehleandrew. cmu. edu (Chieh Lo), kbhardwa@andrew. cmu . edu (Kartikeya Bhardwaj), radum@ cmu . edu (Radu Marculescu)
}

Preprint submitted to Nano Communication Networks have been already proposed. For instance, in [7], the authors propose to design micro-robots with a rotating helical tail in order to prevent invasive drug delivery when swimming in a viscous fluid. However, a more bio-compatible approach is to use bacteria as bio-robots designed to perform pre-determined tasks. A model of using bacterial network to move bacteria toward the target locations has been proposed in [8]; the authors also provide some statistical analysis to quantify the performance of the drug delivery process. Therefore, bacteriabased drug delivery can be a perfect candidate because bacteria can be engineered to navigate to the target location. However, to the best of our knowledge, a mechanism by which bacteria can be engineered to collectively release a precise amount of drugs at the target location, in an adaptive manner has not been developed yet [9]. Consequently, as one of our main contributions, we propose a genetic circuitry that can sense the tumor related signals and then release drugs precisely to the target in an adaptive and coordinated manner.

Getting now into more details, one of the advantages of using bacteria as a drug delivery vehicle is that bacteria can sense multiple types of signals and make decisions through complex regulatory pathways. One such instance is the regulation of bacterial movement known as chemotaxis [10]. In a heterogeneous environment, 


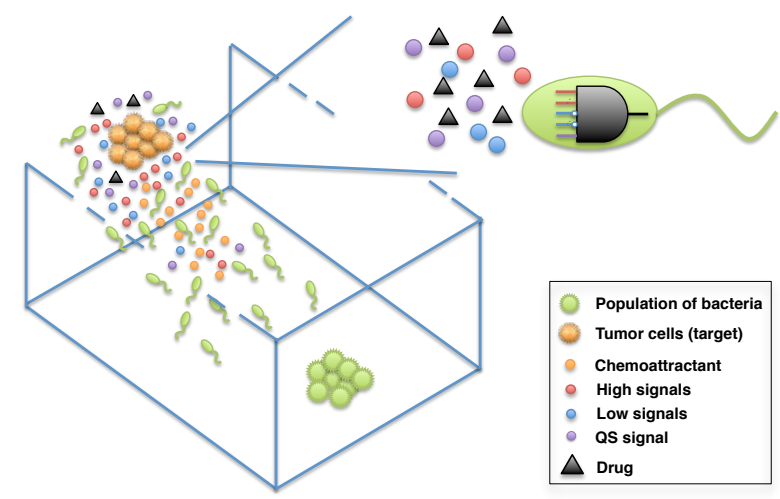

Figure 1: Proposed drug delivery system: The green circles represent clusters of bacteria released at an arbitrary (initial) location. The big orange circles represent the tumor cells that release chemoattractants (shown with small orange circles) into the environment. Bacteria (shown with green) sense the the concentration change of chemoattractants and start accumulating around the tumor. Once bacteria reach a quorum and match the High and Low signals released by the tumor (red and blue particles), the QS signal (purple particles) turns on the drug release circuit (embedded inside the bacteria) which stimulates the production of drugs (black triangles).

bacteria can sense the concentration of the nutrient (i.e., chemoattractants) and control the rotation of the flagella thus deciding the direction of movement [11]. Additionally, it was discovered that tumor cells can release certain types of nutrients that can be recognized by bacteria [8].

Consequently, we propose to utilize bacteria as vehicles to deliver drugs at the target locations where various tumors may be located. The specific receptors for bacterial chemotaxis (anchored on the cell membrane) can bind to chemoattractants; the newly formed complexes can then directly regulate the chemotaxis pathway and drive the movement of bacteria [12]. Therefore, by detecting the concentration gradient of chemoattractants [13], it becomes possible to move and accumulate bacteria at any target location [9, 14]. To effectively transport the drug, we consider a dense network of interacting bacteria that can utilize chemotaxis locally and move in formation. As demonstrated in [8], bacteria can aggregate to form clusters; this can be exploited for precise drug delivery.

In addition to moving bacteria toward a target location, another important issue is the timing of releasing the drugs. A naive approach is to make bacteria release the drugs as soon as they reach the target. However, since not all bacteria can reach the target at the same time, drugs may be released at different times; consequently, the proper drug dosage may actually not be delivered to the target. Hence, it is extremely important

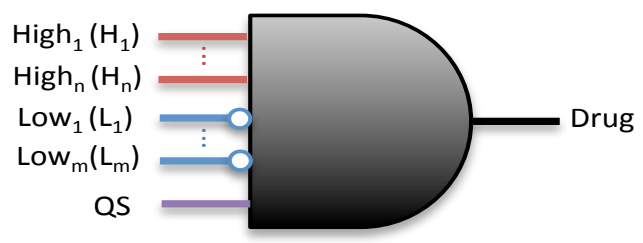

Figure 2: The newly proposed drug release circuitry embedded in the green bacteria in Fig. 1: The multi-input AND gate can sense $n$ High signals, $m$ Low signals, and the QS signal. When the molecular pattern is met, the drug release circuitry starts releasing drug (medicine that targets the tumor cells).

for bacteria to collectively release the drugs only after they all reach the target location. To address this requirement, we propose to utilize a well-known cell-cell communication mechanism [15, 16, 17] known as quorum sensing (QS) [18]; this is needed to coordinate the collective behavior of bacteria. Indeed, by sensing the concentration of specific types of the molecules, bacteria can make decisions in a coordinated fashion, only after reaching a certain cell density threshold [19]. Hence, we propose to integrate the QS signal as a control input to the drug delivery circuitry; Fig. 1 shows our proposed drug delivery system.

Our proposed drug delivery circuitry adaptively controls the amount of drugs being released [20, 21]. As shown in Fig. 2, by recognizing a specific molecular pattern of an active tumor, our proposed genetic circuitry can produce drugs as long as that molecular pattern is matched [22]. Indeed, once the molecular pattern is matched, bacteria start releasing precise amounts of drugs based on the concentration of tumor-related signals. As drugs accumulate and start working, the tumor size starts to decrease, and thus concentration of these molecules decreases ${ }^{1}$. In order to prevent drug resistance, our proposed genetic circuit is able to detect such molecular changes in the environment and dynamically adjust the timing and the amount of drugs released.

In a real-life scenario, however, such as one involving obstruction due to blood vessels, different kinds of chemical substances can block the movement of bacteria. For instance, the extracellular matrix is abundant around tumors [23, 24] and has a higher viscosity compared to the fluid in the environment. Therefore, we need to model such high viscosity regions as obstacles. Once bacteria enter such a region, their speed should significantly decrease. To evaluate the performance of the drug delivery system, we consider the time when

\footnotetext{
${ }^{1}$ We assume that the concentrations of tumor-related signals are proportional to the tumor size.
} 


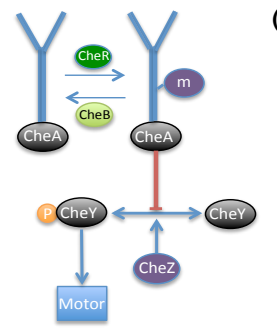

(b)

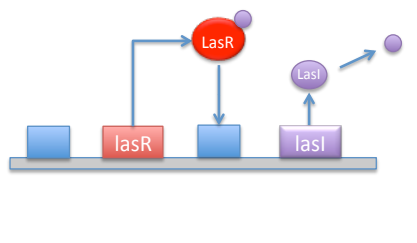

Figure 3: Regulatory pathways of chemotaxis and QS. (a) Bacteria chemotaxis: the blue fork is the signal receptor of the chemoattractant; it can phosphorylate the $C h e Y$ into $C h e Y_{p}$ and decrease the clockwise rotation in order to run. The $C h e R$ and $C h e B$ control the activity of receptor methylation (purple circle $m$ ). The $C h e A$ and $C h e Z$ function as regulators in order to balance the level of $C h e Y$ and $C h e Y_{p}$ (b) QS: the blue box represents the promoter binding regions. QS signaling molecules are expressed by LasI; once the bacterial cell density reaches a certain threshold, QS signaling molecules further combine with LasR receptors that form an positive feedback loop. The purple particles are the same signaling molecules used in Fig. 1

bacteria reach the target location under various conditions (including a varying number of obstacles), obstacles with movements, and different initial distributions of bacteria. The overall performance can be used as a guideline to engineer the drug delivery system.

In terms of design methodology, we first model the chemotaxis and the QS regulatory pathway inside the bacteria. Next, we design the drug delivery genetic circuitry that takes its inputs from the environment (i.e., tumor-released molecular pattern and QS signal). Lastly, we integrate bacterial chemotaxis and drug delivery circuitry to construct an autonomous drug delivery system and demonstrate its effectiveness via detailed simulations [25, 26, 27]. Taken together, our contribution is threefold:

- First, we develop an autonomous drug delivery system that effectively and precisely delivers the appropriate amount of drugs to a target site. To the best of our knowledge, we are the first to combine bacterial chemotaxis and QS signaling in a way that can automatically locate the target and release the drugs in a coordinated manner.

- Second, we propose new synthetic circuitry that can adjust the amount of drugs released based on tumor size and activity. We demonstrate that the proposed AND gate can successfully achieve our goals; this shows the feasibility of the newly proposed autonomous control of bacteria-based drug delivery system.

- Third, we identify the regions of desired operation and characterize the biological parameters of the proposed drug delivery system. The robustness analysis shows that our system can handle a wide range of parameter perturbations. Additionally, we evaluate the spatial effects of both bacteria and obstacles location by using the hitting time to quantify the performance of the drug delivery system. Our experimental results show that such a system can be effectively used for cell-based therapeutics, while preventing drug overuse and multi-drug resistance.

The remainder of this paper is organized as follows. Section 2 presents the mathematical modeling of the drug delivery system. Section 3 presents the experimental setup and results. Section 4 presents the effects of obstacles by considering various configurations of obstacles and bacteria. Finally, conclusion and future work are discussed in Section 5.

\section{Mathematical Modeling of the Drug Delivery Sys- tem}

The proposed drug delivery system consists of two parts: (i) the chemotaxis component guiding bacteria to the target location and (ii) the genetic circuitry needed to coordinate the drug delivery based on QS signals and molecular patterns released by the target tumor. Here, we present the mathematical underpinnings of each component.

\subsection{Bacterial Chemotaxis}

The bacterial chemotaxis regulatory pathway contains several interactions among chemical substances (Fig. 3(a)). The chemical substance that determines the movement of bacteria is the phosphorylated $\mathrm{CheY}^{2}$. Once the concentration of $C h e Y_{p}$ is repressed, bacteria start to run with slight tumbling. Therefore, as long as bacterial receptors (located on the cell membrane) bind to the chemoattractants, they will induce the production of $C h e A$ that can repress $C h e Y_{p}$ and bacteria can move towards the target. Following work in [28], this process can be modeled as follows:

$$
\begin{aligned}
F & =\epsilon_{0}+\log \left(\frac{1+[C] / K_{\text {on }}}{1+[C] / K_{\text {off }}}\right) \\
A & =\frac{1}{1+e^{F}} \\
Y_{p} & =\text { Che }_{\text {tot }} \times A
\end{aligned}
$$

\footnotetext{
${ }^{2}$ Denoted as $\mathrm{Che}_{p}$ in this paper
} 

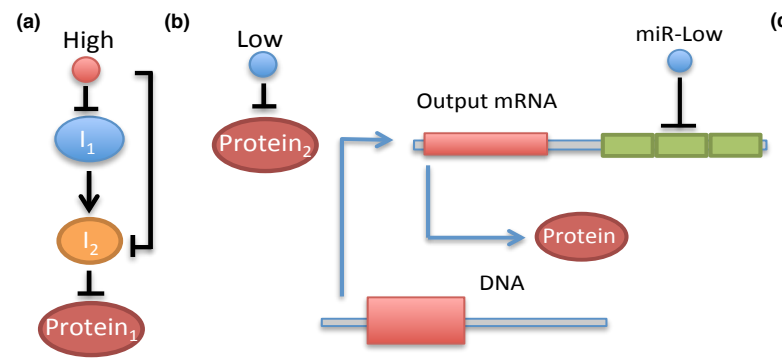

(c)

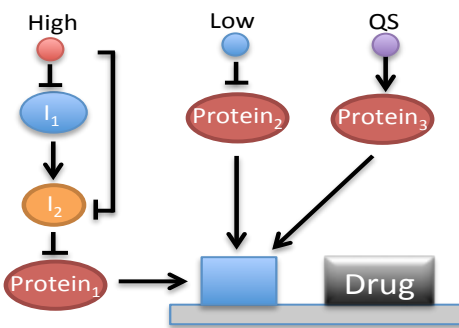

Figure 4: Schematic of the proposed genetic circuitry. (a) The double inversion circuit takes High signals as its input and first represses the intermediate product $l_{1} ; l_{2}$ represses the output protein. (b) The inversion circuit takes Low signals as its input and directly represses the protein (Adapted from [22]). (c) The resulting drug delivery circuitry (Fig. 2) by integrating (a), (b) and QS signal. When the output proteins are all highly expressed, the AND gate starts producing drugs.

where A represents the average activity of the receptors in the cluster, and $F$ is the sum of the energy difference between the receptor on and off states. [C] denotes the concentration of the nutrient, and $C h e Y_{t o t}$ is the total available $C h e Y$ inside the bacteria. $\epsilon_{0}, K_{o n}$ and $K_{o f f}$ are constants. Here, we use the definition of $G_{0}\left(C h e Y_{p}\right), k-$ and $k+$ from reference [29] to determine whether bacteria should run or tumble:

$$
\begin{gathered}
G_{0}\left(\text { Che }_{p}\right)=\frac{g_{0}}{4}-\frac{g_{1}}{2}\left(\frac{C h e Y_{p}}{K_{D}+C h e Y_{p}}\right) \\
k^{+}=w_{0} \exp \left(G_{0}\left(C h e Y_{p}\right)\right) \\
k^{-}=w_{0} \exp \left(-G_{0}\left(C h e Y_{p}\right)\right)
\end{gathered}
$$

where $k^{+}$and $k^{-}$denote the transition rates from clockwise $(\mathrm{CW})$ to counter-clockwise (CCW), and $\mathrm{CCW}$ to $\mathrm{CW}$, respectively; parameters $w_{0}, g_{0}, g_{1}$ are all constants. When flagella turn CCW, bacteria swim straight (this is a run), whereas when they turn $\mathrm{CW}$, bacteria tumble [20]. Using chemotaxis, bacteria can swim and locate the tumor.

\subsection{Bacterial Quorum Sensing (QS)}

We adopt an ODE-based model for a canonical QS las system. The regulatory network of the QS las system has a feedback loop. As shown in Fig. 3(b), the LasR-AI complex up regulates the expression of both lasR and lasI genes. Based on the ODE-models proposed in [30,31], we have the following equations for the las QS system:

$$
\begin{aligned}
& \frac{d[A]}{d t}=c_{A}+\frac{k_{A}[C]}{K_{C}+[C]}-k_{0}[A]-k_{1}[R][A]+k_{2}[R A] \\
& \frac{d[R]}{d t}=c_{R}+\frac{k_{R}[C]}{K_{C}+[C]}-k_{3}[R]-k_{1}[R][A]+k_{2}[R A]
\end{aligned}
$$

$$
\begin{aligned}
\frac{d[R A]}{d t} & =k_{1}[R][A]-k_{2}[R A]-2 k_{4}[R A]^{2}+2 k_{5}[C] \\
\frac{d[C]}{d t} & =k_{4}[R A]^{2}-k_{5}[C]
\end{aligned}
$$

where $[X]$ denotes the concentration of a particular molecular species $X$. In our formulation, $A$ stands for $A I, R$ is LasR, RA is the LasR - AI complex and $C$ is the dimerized complex. $c_{A}$ and $c_{R}$ account for the basal level transcription of $A$ and $R$, respectively. The model parameters of the QS system are shown in Table 1.

\begin{tabular}{|l|l|l|}
\hline Representation & Parameters & Value \\
\hline Basal production rate & $c_{A}, c_{R}$ & $1 \times 10^{-4}$ \\
\hline Maximum production rate & $k_{A}, k_{R}$ & $2 \times 10^{-3}$ \\
\hline Michaelis-Menten constant & $K_{A}, K_{R}$ & $1 \times 10^{-6}$ \\
\hline Association (dissociation) rate & $k_{0}, k_{3}$ & $1 \times 10^{-2}$ \\
\hline Association (dissociation) rate & $k_{1}, k_{2}, k_{4}, k_{5}$ & $1 \times 10^{-1}$ \\
\hline Basal production rate & $r_{0}$ & $1 \times 10^{-3}$ \\
\hline Degradation rate & $\delta$ & $2.5 \times 10^{-1}$ \\
\hline Promoter strength & $P$ & $1 \times 10^{2}$ \\
\hline Michaelis-Menten constant & $K_{H}, K_{Q S}$ & $1 \times 10^{-2}$ \\
\hline
\end{tabular}

Table 1: Model parameters for QS and drug delivery circuitry [31].

\subsection{Drug Delivery Circuitry}

Bacteria can reach a quorum once the cell density reaches the activation threshold; this way once bacteria reach the target location and reach a quorum, the drug delivery circuitry starts releasing the drugs based on the concentration of the tumor related signal. Tumor cells can release a particular molecular pattern that distinguishes one tumor from another. Therefore, we integrate QS signal and this specific pattern as inputs to the drug delivery circuitry.

As shown in Fig. 2, we assume that the tumor cells show a molecular profile that contains $n$ different High 

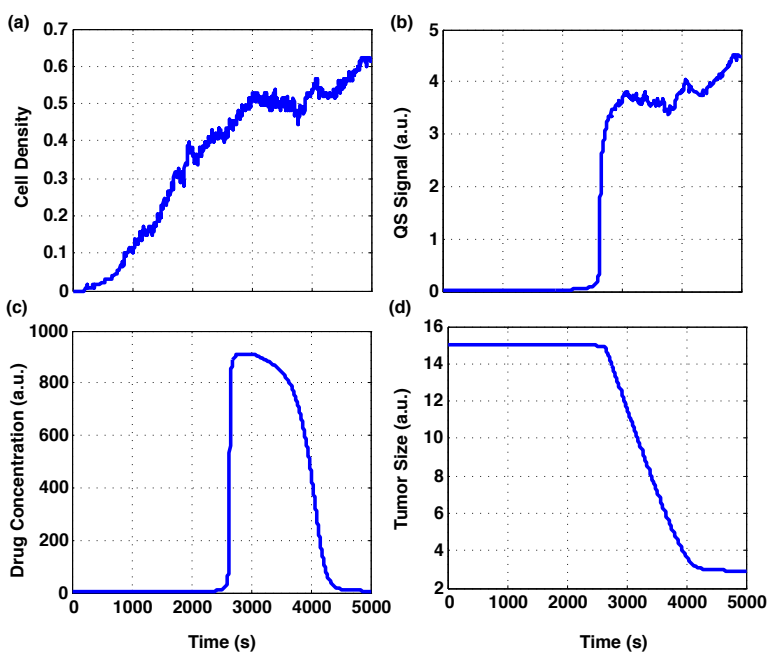

Figure 5: Drug delivery system responses. (a) Bacteria cell density variation: At time of $2500 \mathrm{~s}$, bacteria reach a cell density value around 0.4. (b) QS signal: Bacteria start to release QS signal once the cell density reaches 0.4 (around 2500s). (c) and (d) Drug concentration and tumor size variation. QS signal activates the drug delivery circuitry. As tumor size decreases, the concentration of drugs released by the circuitry also decrease in order to precisely control the amount of drugs. This prevents drug overuse and longer term drug resistance.

$\left(H_{1: n}\right)$ concentration molecules, $m$ Low $\left(L_{1: m}\right)$ concentration molecules, and a QS signal. For example, the molecular pattern in [22] targets the Hela cell by recognizing its particular molecular pattern (Hela-High and Hela-Low signals).

We integrate these three different types of chemical signals into a synthetic AND gate since drugs need to be released only when all the molecular patterns are met. We use a double inversion circuit to take in $H_{1: n}$ as inputs and produce intermediate signals that induce the production of the drug (Fig. 4(a)). The double inversion circuit is needed because the concentrations of $H_{1: n}$ are too low to directly activate the drug circuitry. Hence, the intermediate step of the double inversion circuit serves as a buffer meant to amplify the input concentration. For the low concentration signal $L_{1: m}$, we use a simple inversion circuit (Fig. 4(b)) to invert the input and produce the intermediate signal.

A general inversion genetic circuit can be described by the following equations [32]:

$$
\begin{aligned}
\frac{d[m R N A]}{d t} & =r_{0}-\delta[m R N A]-[Y] \frac{V[m R N A]}{K+[m R N A]} \\
\frac{d[\text { Protein }]}{d t} & =P[m R N A]-\delta[\text { Protein }]
\end{aligned}
$$

where $[X]$ denotes the concentration of a particular

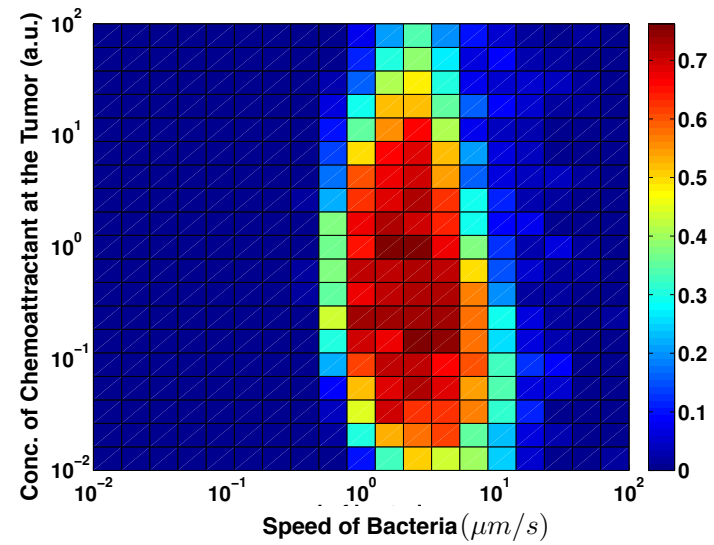

Figure 6: System robustness evaluation using bacteria speed and chemical gradient as variables. The color bar represents the cell density. Green and red colors indicate feasible regions where cell density exceeds the activation threshold of the drug release circuitry (we design the activation threshold to be 0.4 )

molecular species, $Y$ represents the molecules released by the tumor which serves as the input to the genetic circuit ( $H_{1: n}$ or $L_{1: m}$ in Fig. 4$)$. Protein represents the concentration of a certain kind of protein that can trigger the production of drugs. $P$ and $r_{0}$ represent the promoter strength and basal production rate, respectively. Finally, $\delta$ is the degradation constant.

Eq. (10) describes the transcription of mRNA which is directly regulated by the concentration of the input molecules through the Michaelis-Menten kinetics. Eq. (11) describes the translation of the mRNA into protein with the direct regulation of the mRNA.

The QS regulation system produces the QS molecules needed to set the AND gate output to 1 (see Fig. 4(c)). By combining these three types of signals into a synthetic AND gate, we propose the following equation:

$$
\frac{d[D]}{d t}=P\left(\frac{K_{H}}{K_{H}+[H]}\right)^{n_{H}}[L]^{n_{L}} \frac{[Q S]}{[Q S]+K_{Q S}}-\delta[D]
$$

where $D$ represents the concentration of the drug, $L$ is the concentration of the Low signal, $H$ is the concentration of High signal and $Q S$ is the concentration of the QS signal. $K$ is the disassociation constant and the subscript ( $H$ and $L$ ) corresponds to each type of species. $n$ denotes number of types of molecules for both the categories $(H$ and $L)$. In our case, the molecular release profile of the target tumor can be recognized as one repressor and two activators. Hence, $n_{L}$ equals one and $n_{H}$ is two. 

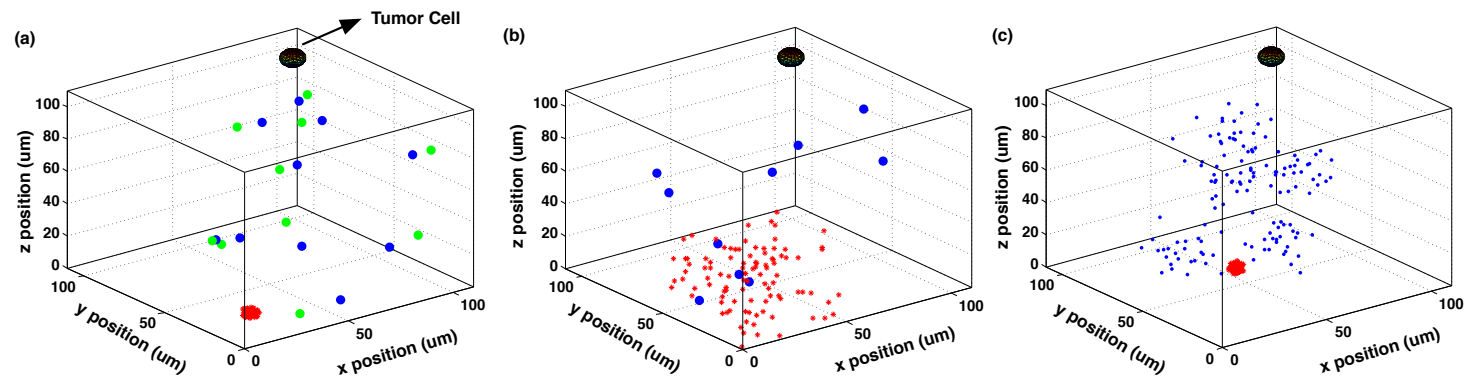

Figure 7: Different simulation configurations. (a) Bacteria clustered at the origin and large size obstacles randomly distributed in space. Blue circles represent (initial) spatial distribution of obstacles, while green circles represent the moving obstacles after certain timestamps. (b) Bacteria randomly distributed. (c) Small size obstacles distributed based on a Gaussian distribution.

\section{Simulation without Obstacles}

In this section, we first describe the simulation environment and parameters for the drug delivery system. Next, we describe the simulation results with respect to both chemotaxis and drug delivery.

\subsection{Simulation Setup and Parameters}

We consider a 3D lattice with length $200 \mu \mathrm{m}$, width $200 \mu \mathrm{m}$, and height $200 \mu \mathrm{m}$. All bacteria (with their initial swimming directions randomly generated) are released from the transmitter group located at $(50,50,50)$ $\mu \mathrm{m}$ of the $3 \mathrm{D}$ space. Of note, we assume bacteria can release chemoattractant locally area and utilize this local attraction force to move in formation.

In the following simulations, the local group size of bacteria is set to 50 . Under these conditions, bacteria swim towards the target location (an aggregate of tumor cells) located in the center of the 3D space, i.e., (100, $100,100) \mu \mathrm{m}$. The tumor cells start emitting chemoattractants in the environment when bacteria are released. Bacteria swim with a constant speed of $10 \mu \mathrm{m} / \mathrm{s}$ [8]. The parameters for QS and drug release circuit are shown in Table 1.

\subsection{Drug Release Circuitry}

We implemented a four-input AND gate including two High signals $\left(H_{1}\right.$ and $\left.H_{2}\right)$, one Low signal $\left(L_{1}\right)$, and one QS signal. At time $t=0 \mathrm{~s}$, we place 5000 bacteria (100 groups each with 50 bacteria) at the origin (i.e., $(0,0,0)$ in $3 \mathrm{D}$ coordinates) while the tumor is located at location $(100,100,100)$; bacteria then use chemotaxis to reach the target site.

We measure the cell density as the ratio of the total volumes occupied by the bacteria divided by the total volume around the tumor ${ }^{3}$. Fig. 5(a) shows the cell density in the vicinity of the tumor as a function of time. We design the activation threshold around 0.4 to trigger the drug release circuitry. For our experiments, the cell density reaches the threshold of 0.4 at time $t=2500 \mathrm{~s}$.

Fig. 5(b) shows the QS signal switching on once the cell density goes above the threshold; this change can be attributed to change in concentration of extracellular QS signaling molecules. Further, because the tumor starts at its full size initially, we assume that the initial concentration of $H_{1}$ and $H_{2}$ equals to 1 and $L_{1}$ is 0 . The output of the AND gate will change from low to high at time $t=2500 \mathrm{~s}$. This is illustrated in Fig. 5(c).

We observe that bacteria do not release drugs as soon as they reach the target site, rather they wait until they reach a quorum and then collectively release the drug until the tumor size is reduced. This coordinated behavior of bacteria makes the drug release protocol more effective. This also shows how the circuit automatically connects the chemotaxis module with the drug release protocol: it waits until the majority of bacteria reach the tumor using chemotaxis and only then generates a response.

Finally, Fig. 5(d) shows the reduction in tumor size due to the drug. As the tumor size decreases, we assume the concentration of molecules released by the tumor also decreases which serves as a feedback to the drug delivery circuitry (Fig. 2); in turn, this reduces the amount of drug being released. This effect can be easily observed from Fig. 5 (d). The drug concentration gradually reduces to zero as the tumor size becomes negligible. Intuitively, this means that our drug delivery system releases precise quantities of the drug and hence prevents any kind of drug resistance or drug overuse.

\footnotetext{
${ }^{3}$ The range of cell density is within [0 1$]$.
} 

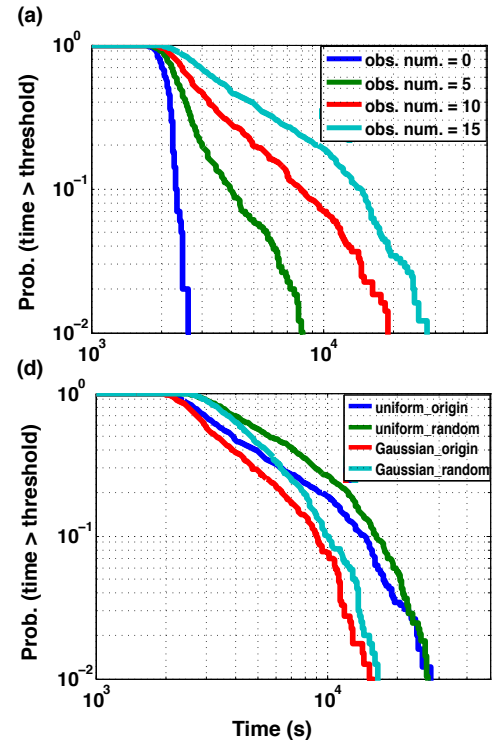

(b)

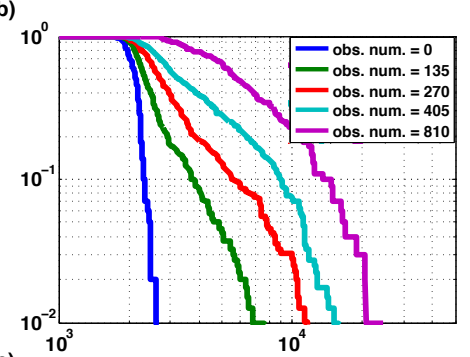

(e)

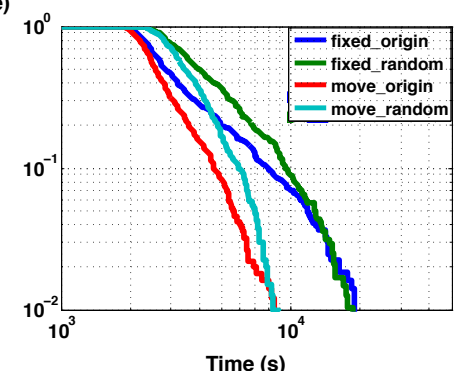

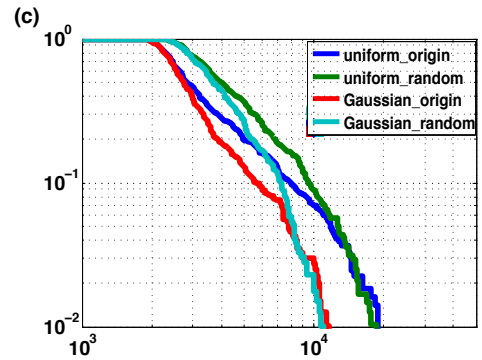

(f)

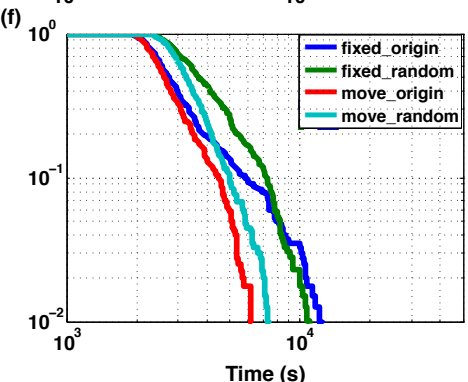

Figure 8: The probability distribution of hitting time to exceed a certain threshold as a function of different configurations. We consider the impact of obstacles number, spatial distribution of obstacles and bacteria, and the movement of bacteria. (a) Obstacle numbers equal to 5 , 10 and 15 and follow uniform distribution. (b) Obstacle numbers equal to 135, 270, 405 and 810 and follow a Gaussian distribution. For both (a) and (b), bacteria are initially located at the origin. (c) and (d) Bacteria with two different initial spatial distributions (located at the origin or randomly distributed). Obstacles follow two different spatial distributions (uniform and Gaussian) under same spatial coverage. (e) and (f) Obstacle number equals to 10 (under uniform distribution) and 270 (under Gaussian distribution), respectively. For both (e) and (f), bacteria are either located at the origin or randomly distributed and obstacles either move or remain fixed.

\subsection{System Robustness}

Characterizing the robustness of the system is crucial since the uncertainty in biological parameters and the stochastic behavior of bacteria can cause undesirable system responses. Hence, we need to determine the range of biological parameters for which the drug delivery system can function well. Note that the drug delivery system can not deliver the drug if the bacteria do not reach a certain cell density needed to activate the QS system. Therefore, the bacterial chemotaxis plays a critical role and should be examined carefully.

We first focus on robustness analysis of the chemotaxis system. We assume that the amount of bacteria released at the origin is large enough and, thus, the meantrajectories of bacteria can represent the movement of the entire population. There are two major factors that can affect the bacterial chemotaxis. One is the intrinsic speed of bacteria movement; the other is the concentration gradient of the chemoattractant. Hence, we vary the value of these two parameters and observe different combinations that can affect the system robustness.

As shown in Fig. 6, the cell density can reach values above the activation threshold of the QS when these two variables are within a certain range (the red region of Fig. 6). It is worth noticing that the speed of bacteria plays a key role compared to the concentration gradient. When the speed of bacteria is too high, bacteria first follow the concentration gradient. However, after several steps, bacteria can not sense the concentration difference of chemoattractants due to their high speed; this results in random trajectories. On the contrary, if the speed of bacteria is too low, it may take a longer time for bacteria to reach the target. Hence, this is not efficient from the drug delivery standpoint.

\section{Simulation with Obstacles}

\subsection{Simulation setup}

In this section, we examine the effect of adding multiple obstacles to the simulation environment. This is a realistic scenario to consider since obstacles such as tissues and extracellular matrix are common in blood vessels. Extracellular matrix, for instance, can trap bacteria due to its high viscosity. Therefore, we assume that if bacteria enter in a region with obstacles, their moving speed is significantly reduced.

We consider two different sizes of obstacles, namely, obstacles as large as the tumor (i.e., $30 \mu m \times 30 \mu m \times$ 30 $\mu \mathrm{m}$ ) (Fig. 8(a) and (b)) and small obstacles of size $10 \mu m \times 10 \mu m \times 10 \mu m$ (Fig. 8(c)). We assume that within 


\begin{tabular}{|c|c|c|c|c|}
\hline Scenario & Average of hitting times (s) & Variance of hitting times & Skewness of hitting times & Kurtosis of hitting times \\
\hline$(0$, Not Applicable, O) & $2.130 \times 10^{3}$ & $3.257 \times 10^{4}$ & 0.269 & 3.543 \\
\hline \hline$(5, \mathrm{~F}, \mathrm{O})$ & $2.795 \times 10^{3}$ & $1.416 \times 10^{6}$ & 2.897 & 12.137 \\
\hline$(10, \mathrm{~F}, \mathrm{O})$ & $4.199 \times 10^{3}$ & $1.100 \times 10^{7}$ & 2.636 & 10.242 \\
\hline$(15, \mathrm{~F}, \mathrm{O})$ & $6.280 \times 10^{3}$ & $2.939 \times 10^{7}$ & 2.003 & 6.945 \\
\hline \hline$(135, \mathrm{~F}, \mathrm{O})$ & $2.697 \times 10^{3}$ & $1.076 \times 10^{6}$ & 3.249 & 16.421 \\
\hline$(270, \mathrm{~F}, \mathrm{O})$ & $3.437 \times 10^{3}$ & $2.833 \times 10^{6}$ & 2.648 & 10.729 \\
\hline$(405, \mathrm{~F}, \mathrm{O})$ & $4.582 \times 10^{3}$ & $8.784 \times 10^{6}$ & 1.820 & 6.258 \\
\hline$(810, \mathrm{~F}, \mathrm{O})$ & $7.437 \times 10^{3}$ & $2.053 \times 10^{7}$ & 1.469 & 4.968 \\
\hline
\end{tabular}

Table 2: Statistical moments of hitting times for different obstacle sizes under the same obstacle distributions. The triplet for each scenario corresponds to the number of obstacles, moving or fixed obstacles, and the spatial distribution of bacteria. "F" means fixed obstacles and "O" represents bacteria clustered at the origin.

\begin{tabular}{|c|c|c|c|c|}
\hline Scenario & Average of hitting times (s) & Variance of hitting times & Skewness of hitting times & Kurtosis of hitting times \\
\hline$(10, \mathrm{~F}, \mathrm{O})$ & $4.199 \times 10^{3}$ & $1.100 \times 10^{7}$ & 2.636 & 10.242 \\
\hline$(10, \mathrm{~F}, \mathrm{R})$ & $5.240 \times 10^{3}$ & $1.104 \times 10^{7}$ & 1.829 & 7.076 \\
\hline$(270, \mathrm{~F}, \mathrm{O})$ & $3.437 \times 10^{3}$ & $2.833 \times 10^{6}$ & 2.648 & 10.729 \\
\hline$(270, \mathrm{~F}, \mathrm{R})$ & $4.341 \times 10^{3}$ & $3.571 \times 10^{6}$ & 1.593 & 5.678 \\
\hline \hline$(15, \mathrm{~F}, \mathrm{O})$ & $6.280 \times 10^{3}$ & $2.939 \times 10^{7}$ & 2.003 & 6.945 \\
\hline$(15, \mathrm{~F}, \mathrm{R})$ & $7.903 \times 10^{3}$ & $3.237 \times 10^{7}$ & 1.470 & 4.637 \\
\hline$(405, \mathrm{~F}, \mathrm{O})$ & $4.582 \times 10^{3}$ & $8.784 \times 10^{6}$ & 1.820 & 6.258 \\
\hline$(405, \mathrm{~F}, \mathrm{R})$ & $5.754 \times 10^{3}$ & $9.831 \times 10^{6}$ & 1.616 & 5.568 \\
\hline
\end{tabular}

Table 3: Statistical moments of hitting times for various spatial distributions of bacteria and obstacles. The triplet for each scenario corresponds to the number of obstacles, moving or fixed obstacles, and the spatial distribution of bacteria. "F" means fixed obstacles. "O" and " $\mathrm{R}$ " represent bacteria clustered at the origin and randomly distributed, respectively.

the region with obstacle, the bacteria speed decreases ten times compared to their usual speed $(10 \mu \mathrm{m} / \mathrm{s})$. Additionally, we consider the the obstacles either follow an uniform or a Gaussian distribution in the environment and then quantify the time when a sufficient number of bacteria reach the target location; this represents the hitting time (or first passage time) and can be regarded as a performance metric for a targeted drug delivery.

\subsection{Statistical measurements}

We measure the hitting times for different configurations along with the baseline scenario i.e., without obstacles. We vary: (1) the number of obstacles and their spatial distributions, (2) the initial spatial distribution of bacteria, and (3) the movement of obstacles. More specifically, when the obstacles are of large size, we randomly distribute them based on an uniform distribution; we need a relatively small number of obstacles (namely, 5, 10, 15), in order to cover the entire simulation space. On the other hand, obstacles of small size follow a Gaussian distribution; they need to be more abundant in order to have similar space coverage.

For the initial (spatial) distribution of bacteria, we consider two scenarios: (1) uniform random distribution and (2) clustered (at the origin) distribution. For the dynamics of obstacles, we consider that they are either static (at the initial position) or perform a random walk as a consequence of drifting in the fluid. For each configuration, we estimate the probability that the hitting time exceeds a certain threshold which can be related to the degree of success of the drug delivery system. Fig. 7 shows different simulation configurations.

For completeness, we report in Tables 2, 3 and 4 the average, variance, skewness, and kurtosis of hitting times ${ }^{4}$. From Tables 2, 3 and 4, one can observe that the hitting times exhibit a positive skewness which has two implications: First, the tail of the distribution of hitting time is longer towards the right direction (corresponding to larger hitting times), while towards the left hand side the tail is shorter i.e., shorter hitting times. Second, since the mass of the distribution is concentrated towards the right hand side, one cannot ignore the chances of seeing much larger hitting times.

\subsection{Impact of number of obstacles}

We now quantify the effect of obstacles number. From Table 2, it can be observed that the average hitting times increase when the number of obstacles increases (from $20 \%$ to $160 \%$ ), if all other conditions are fixed. We notice that with a greater number of obstacles, the probability of having very large hitting times gets larger (see Fig. 8(a) and (b)). However, the distribution of hitting times is less skewed; This is because the distribution does not have apparent peaks and is more similar

\footnotetext{
${ }^{4}$ The skewness and kurtosis reflect the asymmetry in the hitting times distribution.
} 


\begin{tabular}{|c|c|c|c|c|}
\hline Scenario & Average of hitting times $(\mathbf{s})$ & Variance of hitting times & Skewness of hitting times & Kurtosis of hitting times \\
\hline$(10, \mathrm{~F}, \mathrm{O})$ & $4.199 \times 10^{3}$ & $1.100 \times 10^{7}$ & 2.636 & 10.242 \\
\hline$(10, \mathrm{M}, \mathrm{O})$ & $3.045 \times 10^{3}$ & $1.722 \times 10^{6}$ & 2.660 & 12.857 \\
\hline \hline$(10, \mathrm{~F}, \mathrm{R})$ & $5.240 \times 10^{3}$ & $1.104 \times 10^{7}$ & 1.829 & 7.076 \\
\hline$(10, \mathrm{M}, \mathrm{R})$ & $3.920 \times 10^{3}$ & $2.089 \times 10^{6}$ & 1.909 & 8.931 \\
\hline \hline$(270, \mathrm{~F}, \mathrm{O})$ & $3.437 \times 10^{3}$ & $2.833 \times 10^{6}$ & 2.648 & 10.729 \\
\hline$(270, \mathrm{M}, \mathrm{O})$ & $2.954 \times 10^{3}$ & $9.449 \times 10^{5}$ & 1.650 & 5.592 \\
\hline \hline$(270, \mathrm{~F}, \mathrm{R})$ & $4.341 \times 10^{3}$ & $3.571 \times 10^{6}$ & 1.593 & 5.678 \\
\hline$(270, \mathrm{M}, \mathrm{R})$ & $3.574 \times 10^{3}$ & $1.184 \times 10^{6}$ & 1.718 & 6.344 \\
\hline
\end{tabular}

Table 4: Statistical moments of hitting times for the effects of obstacle movements. The triplet for each scenario corresponds to the number of obstacles, moving or fixed obstacles, and the spatial distribution of bacteria. "F" represents fixed obstacles at the initial location, while "M" indicates obstacles perform random walks. "O" and " $\mathrm{R}$ " represent bacteria clustered at the origin and randomly distributed, respectively.

to a Gaussian distribution. On the contrary, for the scenario of less obstacles, the distribution of hitting times is more skewed and has a longer tail; therefore, the probability of having larger hitting times is lower.

We also consider a scenario in which obstacles number is huge and cover the space up to $40 \%$. As shown in Fig. 8(b), the purple line (obstacles number equal to 810) has a less skewed hitting times distribution but the average hitting times is about two times longer when space coverage reduces to $20 \%$.

\subsection{Impact of spatial distribution of obstacles}

As discussed above, small size obstacles are assumed to follow a Gaussian distribution. The mean of the Gaussian distribution is randomly generated and the standard deviation is chosen to match the size of larger obstacles in order to have same space occupations. As shown in Table 3 and Fig. 8(c) and (d), uniformly distributed obstacles (i.e., larger sizes) have similar average and skewness of hitting times when other conditions are fixed. This shows that the spatial distribution of obstacles has little effect on the distribution of hitting times which results from similar spatial coverages of obstacles even if they follow different spatial distributions.

\subsection{Impact of spatial distribution of bacteria}

In this section, we investigate the impact of spatial distribution of groups of bacteria in the simulation space. A group of around 50 bacteria is formed by the local forces resulting from the chemoattractants expressed by other bacteria. To uniformly distribute bacteria in space, one can utilize repulsion forces to separate groups of bacteria; this can be achieved by engineering the bacteria regulatory pathway of chemotaxis to respond to a certain kind of chemorepllent. However, the chemo-repulsion forces among clusters of bacteria should be weaker than the chemo-attraction force within the group because we need to ensure the separation of groups of bacteria while still maintaining the local clustering. After arriving at the target location, the chemo-repulsion forces should be stopped by switching off the regulatory pathway of the chemotaxis.

As shown in Table 3 and Fig. 8(c) and (d), in general, different spatial distributions of bacteria have a similar performance in terms of average of hitting times. The main difference is in the skewness of hitting times. The randomly distributed case has a lower skewness because the probability of bumping into obstacles is lower than clustered (at the origin) distribution; this implies that the probability of successfully delivering drugs is higher when bacteria are randomly distributed in the space.

Additionally, when bacteria initially clustered at the origin of the 3D space, the distribution of hitting times exhibits heavy tails. However, the baseline scenario shows that bacteria start from the origin have a better performance than the randomly distributed one; this implies that obstacles degrade the performance of drug delivery system heavily.

\subsection{Impact of moving obstacles}

We assume now that the movement of obstacles follows a random walk i.e., obstacles move back and forth around the initial locations; this property results in shorter average values of hitting times since the probability of trapping bacteria within the obstacle location decreases.

As can be seen in Table 4 and Fig. 8(e) and (f), when obstacles move freely in the simulation space (other conditions being fixed), the average of hitting times decreases compared to the scenario of fixed obstacle locations; this is because the obstacles can no longer trap bacteria and bacteria regain the normal speed to move toward the target location. However, the movement of obstacles has little effect in terms of the skewness of hitting times. Hence, as shown in Fig. 8(e) and (f), the shapes of the hitting time distributions for each scenario are similar. 


\subsection{Summary of results derived in the presence of ob- stacles}

As a summary of these investigations, the presence of obstacles increases the hitting times in the range of $20 \%$ to $160 \%$; this greatly affects the overall drug delivery performance. In general, when the number of obstacles increases, the average of hitting times increases too because of the probability of bumping into obstacles also gets higher.

We also find that the spatial distribution of obstacles has indistinguishable effects on the distribution of hitting times. On the contrary, randomly distributed bacteria can have better performance in terms of the skewness of hitting times. Finally, moving obstacles can decrease the average of hitting times since the probability of trapping bacteria decreases.

Overall, the presence of obstacles degrades the performance of our proposed drug delivery system. Our complete analyses above can provide strategies to enhance the performance of the drug delivery system. For example, since randomly distributed bacteria have better performance in terms of hitting times, we can engineer bacterial chemotaxis to achieve this desired goal.

\section{Conclusions and Future Work}

In this paper, we have proposed a cell-based therapeutic approach that utilizes bacteria to deliver drugs autonomously and adaptively. To this end, we have designed a new drug delivery system such that it automatically integrates the chemotaxis and QS signals. We have further demonstrated the functionality of the drug delivery system that can work in an adaptive manner and reduce the tumor size dynamically. We have also examined how the environmental variables, obstacles, and other factors affect the performance of drug delivery system.

Finally, we have shown that our proposed system satisfies two of the most important characteristics any drug delivery system should have, namely (i) locate the target precisely, and (ii) deliver precise quantities of drugs that decrease as the size of tumor reduces. This is not the case in the conventional drug delivery systems that use diffusion to transport drugs to any given location. Therefore, the proposed drug delivery system prevents unnecessary drug overuse and multi-drug resistance. Our simulation framework can help the synthetic biologists design such bacteria-based drug delivery systems.

\section{References}

[1] S. H. Jang, M. G. Wientjes, D. Lu, J. L.-S. Au, Drug delivery and transport to solid tumors, Pharmaceutical Research 20 (9) (2003) 1337-1350

[2] O. Trédan, C. M. Galmarini, K. Patel, I. F. Tannock, Drug resistance and the solid tumor microenvironment., Journal of the National Cancer Institute 99 (19) (2007) 1441-54.

[3] I. Tannock, C. Lee, J. Tunggal, Limited penetration of anticancer drugs through tumor tissue a potential cause of resistance of solid tumors to chemotherapy, Clinical cancer 8 (March) (2002) 878-884.

[4] N. Forbes, Engineering the perfect (bacterial) cancer therapy, Nature Reviews Cancer 10 (11) (2010) 785-794.

[5] W. D. Joo, I. Visintin, G. Mor, Targeted cancer therapy-are the days of systemic chemotherapy numbered?, Maturitas 76 (4) (2013) 308-14.

[6] A. A. Alexander-Bryant, W. S. Vanden Berg-Foels, X. Wen, Bioengineering strategies for designing targeted cancer therapies., 1st Edition, Vol. 118, Elsevier Inc., 2013.

[7] F. Z. Temel, A. G. Erman, S. Yesilyurt, Characterization and modeling of biomimetic untethered robots swimming in viscous fluids inside circular channels, IEEE/ASME Transactions on Mechatronics 19 (5) (2014) 1562-1573.

[8] G. Wei, P. Bogdan, R. Marculescu, Bumpy rides: Modeling the dynamics of chemotactic interacting bacteria, IEEE Journal on Selected Areas in Communications 31 (12) (2013) 879-890.

[9] Y. Chahibi, I. Akyildiz, S. Balasubramaniam, Y. Koucheryavy, Molecular Communication Modeling of Antibodymediated Drug Delivery Systems., IEEE transactions on bio-medical engineering 00 (00) (2015) 1-13. doi:10.1109/TBME.2015.2400631.

[10] Y. Okaie, T. Nakano, T. Hara, S. Nishio, Target detection and tracking by bionanosensor networks, SpringerBriefs in computer science.

[11] L. E. Weiss, J. P. Badalamenti, L. J. Weaver, A. R. Tascone, P. S. Weiss, T. L. Richard, P. C. Cirino, Engineering motility as a phenotypic response to LuxI/R-dependent quorum sensing in Escherichia coli., Biotechnology and bioengineering 100 (6) (2008) 1251-5.

[12] R. W. Kasinskas, N. S. Forbes, Salmonella typhimurium lacking ribose chemoreceptors localize in tumor quiescence and induce apoptosis., Cancer research 67 (7) (2007) 3201-9.

[13] H.-C. Wu, C.-Y. Tsao, et al, Autonomous bacterial localization and gene expression based on nearby cell receptor density., Molecular systems biology 9 (636) (2013) 636.

[14] B. J. Toley, N. S. Forbes, Motility is critical for effective distribution and accumulation of bacteria in tumor tissue., Integrative biology : quantitative biosciences from nano to macro 4 (2) (2012) $165-76$.

[15] L. C. Cobo, I. F. Akyildiz, Bacteria-based communication in nanonetworks, Nano Communication Networks 1 (4) (2010) 244-256.

[16] T. Nakano, et al., Molecular Communication, Cambridge University Press.

[17] I. F. Akyildiz, F. Brunetti, C. Blázquez, Nanonetworks: A new communication paradigm, Computer Networks 52 (12) (2008) 2260-2279.

[18] B. L. Bassler, R. Losick, Bacterially speaking., Cell 125 (2) (2006) 237-46

[19] A. Jayaraman, T. Wood, Bacterial quorum sensing: signals, circuits, and implications for biofilms and disease, Annu. Rev. Biomed. Eng. 10 (2008) 145-167.

[20] M. A. Roberts, A. Papachristodoulou, J. P. Armitage, Adaptation and control circuits in bacterial chemotaxis., Biochemical Society transactions 38 (5) (2010) 1265-9. 
[21] S. Topp, J. Gallivan, Guiding bacteria with small molecules and RNA, Journal of the American Chemical Society 30322 (15) (2007) 6807-6811.

[22] Z. Xie, L. Wroblewska, L. Prochazka, Multi-input RNAi-based logic circuit for identification of specific cancer cells, Science 333 (2011) 1307-1312.

[23] P. Hinow, P. Gerlee, A spatial model of tumor-host interaction application of chemotherapy, Mathematical biosciences and engineering 6 (3) (2009) 521-546.

[24] N. R. Raz, M. R. Akbarzadeh-T., M. Tafaghodi, Bioinspired nanonetworks for targeted cancer drug delivery, IEEE Transactions on NanoBioscience 14 (8) (2015) 894-906.

[25] G. Wei, P. Bogdan, R. Marculescu, Efficient Modeling and Simulation of bacteria-based Nanonetworks with BNSim, IEEE Journal on Selected Areas in Communications 31 (12) (2013) 868-878.

[26] C. Lo, G. Wei, R. Marculescu, Towards Autonomous Control of Molecular Communication in Populations of Bacteria, Proceedings of the 2nd ACM International Conference on Nanoscale Computing and Communication - NANOCOM'15 (2015) 1-6.
[27] C. Lo, K. Bhardwaj, R. Marculescu, An Autonomous and Adaptive Bacteria-based Drug Delivery System, Proceedings of the 3rd ACM International Conference on Nanoscale Computing and Communication - NANOCOM'16 (2016) 1-6.

[28] L. Jiang, Q. Ouyang, Y. Tu, Quantitative modeling of Escherichia coli chemotactic motion in environments varying in space and time., PLoS computational biology 6 (4) (2010) e1000735.

[29] M. W. Sneddon, J. R. Faeder, T. Emonet, Efficient modeling, simulation and coarse-graining of biological complexity with NFsim., Nature methods 8 (2) (2011) 177-83.

[30] J. W. Williams, X. Cui, A. Levchenko, A. M. Stevens, Robust and sensitive control of a quorum-sensing circuit by two interlocked feedback loops., Molecular systems biology 4 (234) (2008) 234.

[31] P. Melke, P. Sahlin, A. Levchenko, H. Jönsson, A cell-based model for quorum sensing in heterogeneous bacterial colonies., PLoS computational biology 6 (6) (2010) e1000819.

[32] C. A. Voigt, Genetic parts to program bacteria., Current opinion in biotechnology 17 (5) (2006) 548-57. 